gene (SMN1). Copies of a similar gene (SMN2) modify disease severity. In a phase 1 study, SMN GRT onasemnogene abeparvovec (AVXS-101) improved outcomes of symptomatic SMA patients with two SMN2 copies (2xSMN2) dosed $\leq 6$ months. Because motor neuron loss can be insidious and disease progression is rapid, early intervention is critical. This study evaluates AVXS-101 in presymptomatic SMA newborns.

Methods SPR1NT is a multicenter, open-label, phase 3 study (NCT03505099) enrolling $\geq 27$ SMA patients with 2$3 \times$ SMN2. Asymptomatic infants $\leq 6$ weeks receive a one-time intravenous AVXS-101 infusion $\left(1.1 \times 10^{14} \mathrm{vg} / \mathrm{kg}\right)$. Safety and efficacy are assessed through study end (18 [2xSMN2] or 24 months [3xSMN2]). Primary outcomes: independent sitting for $\geq 30$ seconds (18 months [2xSMN2]) or assisted standing (24 months [3xSMN2]).

Results From April-September 2018, 7 infants received AVXS-101 (4 female; 6 with 2xSMN2) at ages 8-37 days. Mean baseline Children's Hospital of Philadelphia Infant Test of Neuromuscular Disorders (CHOP-INTEND) score was $41.7(\mathrm{n}=6)$, which increased by $6.8,11.0,18.0$, and 22.5 points at day $14(n=4)$, month $1(n=3), 2(n=3)$, and 3 $(n=2)$. As of January 31, 2019, 15 asymptomatic infants have been enrolled in SPR1NT and dosed with AVXS-101. Updated data available at the time of the congress will be presented.

Conclusions Preliminary data from SPR1NT show rapid motor function improvements in presymptomatic SMA patients.

\section{UNRAVELLING PSYCHOSIS IN MOTOR NEURONE DISEASE - A STUDY OF CLINICAL FEATURES, COGNITION, AND SURVIVAL}

Emma M Devenney*, Rebekah M Ahmed, Jashelle Caga, Elizabeth Highton-Williamson, Eleanor Ramsey, Margaret Zoing, John Hodges, Matthew Kiernan. University of Sydney, Camperdown, NSW, Australia

\subsection{6/jnnp-2019-anzan.15}

Introduction Psychotic symptoms are now recognised to occur in patients with MND, often in association with FTD, and particularly in C9orf72 expansion carriers. As yet the impact of these symptoms on the clinical disease state is unknown and the relationship between severity and nature of these symptoms is not well understood. This study aimed to comprehensively explore the relationship between psychotic symptoms, clinical features, cognitive status and survival.

Methods In total 148 participants; MND $(n=100)$ and MNDFTD $(n=48)$, were enrolled in the study. A detailed clinical interview in addition to a neurological, neuropsychological and behavioural assessment, genetic testing and brain MRI was undertaken in each participant

Results Psychotic symptoms were present in $25 \%$ of the cohort. The majority of participants in the psychosis cohort were male (83\%) and were negative for the C9orf72 expansion $(70 \%)$. Psychotic symptoms in younger patients were more likely to be florid, require medication and delay diagnosis. Within the MND subgroup, patients with psychotic symptoms were more impaired in the cognitive subdomains of attention, memory and executive functioning and exhibited more disinhibition, apathy and stereotypy, than patients without psychotic symptoms (all $p<0.01$ ), but no differences were identified for the MND-FTD subgroup (all $p>0.2$ ). Symptoms of depression were more common in those without psychotic symptoms $(p>0.1)$. Survival was prolonged for patients with psychotic symptoms $(\mathrm{HR}=4.7,95 \%$ CI: $2.1-10, \mathrm{p}<0.001)$

Conclusion MND with psychosis represents a distinct clinical, cognitive and behavioural phenotype that has a positive impact on survival and may represent an overlap with psychiatric disorders.

\section{ONE DISEASE OR THREE: IS FRONTOTEMPORAL DEMENTIA - MOTOR NEURON DISEASE A DISTINCT ENTITY?}

\begin{abstract}
1,2 Zhe (Jill) Long*, 1,3 Muireann Irish, 1,3 David Foxe, 1,2 John Hodges, 1,3 Olivier Piguet, 1,4,5 James Burrell. 'The University of Sydney, Brain and Mind Centre, Camperdown, NSW, Australia; '2Sydney Medical School, The University of Sydney, Sydney, NSW, Australia; ${ }^{3}$ School of Psychology, The University of Sydney, Sydney, NSW, Australia; ${ }^{4}$ Concord Clinical School, The University of Sydney, Sydney, NSW, Australia; ${ }^{5}$ Neurosciences 5 West, Concord General Hospital, Sydney, NSW, Australia
\end{abstract}

\subsection{6/jnnp-2019-anzan.16}

Introduction Frontotemporal dementia-motor neuron disease (FTD-MND) is diagnosed when patients meet criteria for the diagnosis of both FTD and MND, but the mode presentation of this disorder is currently unknown. This study aimed to compare the mode of presentation, and profiles of behavioural and language disturbances, of FTD-MND with that of other FTD phenotypes using a data-driven approach.

Methods 31 FTD-MND, 119 bvFTD, 47 PNFA, 42 SD patients and 127 controls underwent comprehensive clinical, neuropsychological and neuroimaging assessments. Z-transformed scores were used to compare the severity of behavioural and language domains in each disease group. Two-step cluster analysis profiled patient subgroups. Voxel-based morphometry investigated differential patterns of cortical atrophy between groups.

Results Overall, FTD-MND patients presented with behavioural or language disturbances less frequently than FTD phenotypes, but mixed behavioural-language presentations were more common. FTD-MND patients demonstrated less severe disinhibition, apathy and semantic deficits relative to bvFTD and SD respectively.Behavioural and language deficits were of comparable severity in FTD-MND, unlike other FTD phenotypes where behaviour was worse than language (bvFTD) or language worse than behaviour (PNFA, SD).In cluster analysis, FTD-MND patients were evenly distributed across three subgroups designated as 'mild mixed', 'language dominant' and 'behavioural dominant'. Relative to the 'mild mixed' group, 'language dominant' patients demonstarted more atrophy of the anterior temporal lobe and peri-insular regions, while 'behavioural dominant' patients displayed more prefrontal atrophy.

Conclusion FTD-MND does not present as a uniform syndrome. Rather, there may be at least three subgroups that demonstrate distinctive cognitive, behavioural, and neuroanatomical characteristics. 